Punto de vista

\title{
Alcohol y drogas de abuso en patología traumática. Necesidad de screening rutinario para implantación de programas de prevención secundaria
}

\author{
E. FERNÁNDEZ MONDÉJAR
}

Unidad de Cuidados Intensivos del Hospital de Traumatología. Hospital Universitario Virgen de las Nieves. Granada.

\begin{abstract}
El consumo de alcohol y otras sustancias que alteran el comportamiento constituyen el principal factor de riesgo para sufrir traumatismos graves. Los pacientes que han sufrido un accidente relacionado con el consumo de alcohol u otras drogas presentan un elevado riesgo de reincidencia.

Actualmente no se escatiman esfuerzos para conseguir la recuperación física de estos pacientes, sin embargo, a diferencia de otras patologías, los esfuerzos encaminados a prevenir la reincidencia (prevención secundaria) son prácticamente nulos. Una intervención psicológica breve realizada durante el encamamiento puede modificar el comportamiento y puede disminuir el riesgo de nuevos accidentes; por tanto la implantación de programas de prevención secundaria debe considerarse un objetivo prioritario en centros donde se reciben pacientes traumatizados.
\end{abstract}

PALABRAS CLAVE: prevención secundaria, alcohol, trauma.

\section{ALCOHOL AND ABUSE DRUGS IN TRAUMA PATHOLOGY}

Alcohol and other substance abuse that alters behavior make up the principal risk factor to suffer serious traumas. Patients who have had an alcohol or other drug consumption related accident have a high risk of recidivism.

Correspondencia: Dr. E. Fernández Mondéjar.

Unidad de Cuidados Intensivos del Hospital de Traumatología

Hospital Universitario Virgen de las Nieves.

Carretera de Jaén, s/n.

18013 Granada.

Correo electrónico: enrique.fernandez.mondejar.sspa@juntadeandalucia.es

Manuscrito aceptado el 20-XII-2006.
Currently, no efforts are spared to achieve the physical recovery of these patients. However, on the contrary to other diseases, the efforts aimed at preventing recidivism (secondary prevention) are practically null. A brief psychological intervention made during the bedridden period may modify the behavior and decrease the risk of new accidents. Therefore, establishing secondary prevention programs should be considered a priority objective in sites that receive traumatized patients.

KEY WORDS: secondary prevention, alcohol, trauma.

La relación entre consumo de drogas y los accidentes (de tráfico o de cualquier tipo) es sobradamente conocida. En el caso del alcohol, su relación con los traumatismos se conoce desde la antigüedad ${ }^{1}$, y actualmente sigue considerándose el principal protagonista de esta triste plaga ${ }^{2}$. En los últimos 20 años el consumo de otras drogas, en particular el cannabis, la cocaína y las anfetaminas, ha aumentado de forma considerable, lo que hace que, actualmente, el consumo de drogas lícitas e ilícitas constituya el principal factor de riesgo para sufrir traumatismos.

\section{DETECCIÓN DE DROGAS DE ABUSO EN FALLECIDOS POR ACCIDENTE DE TRÁFICO}

En estudios forenses se demuestra que el alcohol suele estar presente en un 30 o $40 \%$ de los fallecidos por accidente de tráfico, seguido a mucha distancia de la cocaína $(5 \%)$ y el cannabis $(2-3 \%)^{3}$. Estos datos concuerdan con los publicados periódicamente por la Dirección General de Tráfico. En 2002 el porcentaje de casos con alcoholemia superior a la tasa permitida 
en conductores fallecidos por accidente de tráfico era del $32 \%$, frente a un $5,5 \%$ en los controles preventivos (conductores analizados de forma aleatoria).

\section{DETECCIÓN DE DROGAS DE ABUSO EN ACCIDENTADOS NO FALLECIDOS}

Hasta ahora no conocíamos ningún dato de nuestro entorno sobre la incidencia de detección de tóxicos en pacientes traumatizados graves pero no fallecidos. Nuestro grupo realizó un estudio piloto con resultados extremadamente preocupantes. El 58\% de los menores de 45 años ingresados por un traumatismo grave dieron resultados positivos a algún tóxico, y el más frecuentemente encontrado fue el cannabis (35\%), seguido por el alcohol $(16 \%)$ y la cocaína $(15 \%)^{4}$. Estas cifras son similares a las encontradas por otros autores en determinadas áreas de Estados Unidos ${ }^{5,6}$, y sugieren un extraordinario incremento de la detección de cannabis y cocaína. No conocemos estudios epidemiológicos en los que se analice el porcentaje de población general en la que se detecta la presencia de tóxicos en sangre u orina, pero las encuestas de prevalencia en el consumo pueden orientarnos en este sentido. En Andalucía, en el último mes, un $8 \%$ de la población reconoce haber consumido cannabis y un $1,6 \%$ cocaína ${ }^{7}$. Si consideramos estas cifras como representativas de la población general los resultados de nuestro estudio piloto indican que dichos datos se disparan en pacientes traumatizados graves, por lo que existe una clara asociación entre accidentes graves y consumo de sustancias estupefacientes.

\section{NECESIDAD DE REALIZAR PREVENCIÓN SECUNDARIA}

Actualmente se considera que el alcohol y otras drogas constituyen el principal factor de riesgo en pacientes traumatizados. Los pacientes que han sufrido un accidente relacionado con el consumo de estas sustancias presentan un elevado riesgo de reincidencia, y las posibilidades de fallecer en otro accidente duplican las de la población normal ${ }^{8}$.

Lo mismo que cuando un paciente sufre un infarto de miocardio debemos investigar la presencia de factores de riesgo (diabetes, hipertensión, hipercolesterolemia, etc.) y aplicar un tratamiento, en el caso de los traumatizados debemos investigar si existe alguna relación con el consumo de alcohol o de otras drogas y plantear actuaciones encaminadas a evitar la reincidencia. Esto que denominamos prevención secundaria, y que es habitual y obligatorio para cualquier patología, en la patología traumática generalmente no se realiza, en ocasiones por ignorancia, pero muchas veces por un mal entendido «respeto a la intimidad». Lo cierto es que en la mayoría de los centros no se analiza sistemáticamente la relación entre drogas y los traumatismos graves. De esta forma se infraestima la magnitud del problema, pero sobre todo se priva a los pacientes de recibir «consejo psicológico» para poder prevenir nuevos accidentes. Es decir, no se realiza ninguna prevención secundaria, y en un sistema en el que no se escatima ningún medio para conseguir la recuperación física del paciente, tenemos la sensación de que devolvemos a la sociedad sujetos que están condenados a repetir su tragedia. Es por tanto necesario el screening rutinario de tóxicos y la implantación de programas de prevención secundaria para evitar la reincidencia9.

\section{¿CÓMO DEBERÍA REALIZARSE LA PREVENCIÓN SECUNDARIA Y EVITAR LA REINCIDENCIA?}

Es necesaria la colaboración con profesionales dedicados al campo de la adicción. Ellos tienen la experiencia necesaria para abordar con más posibilidades de éxito este tipo de situaciones.

En cuanto a qué debe hacerse es difícil generalizar, debido a los diversos tipos de sustancias estupefacientes y al diferente grado de adicción de los pacientes. El alcohol es sin duda el más analizado, y actualmente existen varios estudios que demuestran que una «intervención psicológica breve» puede modificar el comportamiento y disminuye el riesgo de sufrir nuevos traumatismos ${ }^{10,11}$.

Esta intervención psicológica breve consiste en informar al paciente del resultado positivo obtenido en la determinación de tóxicos y del riesgo vital al que ha estado sometido. Asimismo, debe ponerse de relieve que todos estos riesgos pueden tener relación con el consumo de sustancias estupefacientes, el peligro que implica seguir con este comportamiento y la necesidad de modificar su conducta en este aspecto.

\section{¿CUÁNDO REALIZAR LA «INTERVENCIÓN PSICOLÓGICA BREVE»?}

Esta intervención psicológica breve debe realizarse durante el mismo encamamiento, con objeto de aprovechar el efecto psicológico favorable dentro de la situación de convalecencia del accidente. Cambiar el comportamiento de las personas es una tarea complicada, pero si alguna circunstancia puede ayudar es, sin duda, la situación de convalecencia en la que el paciente es consciente del riesgo al que ha estado sometido y del peligro de persistir en esa conducta.

\section{GARANTÍAS DE CONFIDENCIALIDAD}

Una barrera para la determinación de tóxicos es sin duda el temor a invadir la intimidad de los pacientes y que, por tanto, pueda implicar acciones legales posteriores. En este sentido hay que recordar que la realización de este tipo de análisis requiere dos requisitos básicos: a) ha de ser de utilidad directa para el paciente y b) ha de garantizarse la confidencialidad de los resultados.

En cuanto al primer requisito, la utilidad para el paciente, la detección de tóxicos es útil para el paciente tanto a corto como a largo plazo. Una elevada tasa de alcoholemia o la constatación de la presencia de otro estupefaciente pueden orientar hacia una situación de dependencia ${ }^{2}$, alertándonos sobre una po- 
sible patología asociada (afectación hepática, trastornos de coagulación, etc.) o justificando una excesiva tolerancia a diversos medicamentos. Asimismo, puede ayudar a detectar o prevenir un síndrome de retirada. Pero quizás el mayor beneficio para el paciente se obtenga a largo plazo. Es ampliamente conocido que estos sujetos suelen ser «reincidentes», y cuando se profundiza en su historia clínica detectamos que con frecuencia este accidente no es el primero. La «reincidencia» obviamente tiene graves implicaciones (bajas laborales, hospitalizaciones prolongadas, posibles lesiones a terceros, secuelas físicas y psíquicas, etc.), pero además, como antes apuntábamos, estos pacientes presentan un riesgo de fallecer en otro accidente que dobla al del resto de la población ${ }^{5}$. Por este motivo la detección de una posible adicción, y la orientación hacia programas de rehabilitación, supone sin duda un beneficio directo para el paciente. Por todo esto el establecimiento de programas que, garantizando las medidas de confidencialidad, contribuyan a evitar la reincidencia, debe considerarse un deber ético ineludible?.

Dada la magnitud del problema y la evidencia acumulada, el American College of Súrgeons (SubComité de Trauma) ha establecido una resolución en la que se incluye la determinación de tóxicos y la intervención psicológica como un requisito esencial para que un centro sea acreditado como receptor de Traumatizados de Nivel $1^{12}$.

El consumo de alcohol y otras drogas constituye el principal factor de riesgo de traumatismos graves. Los pacientes traumatizados con resultados positivos a tóxicos tienen un elevado riesgo de reincidencia, por lo que la asistencia integral de estos pacientes debe plantearse no sólo en términos de conseguir la mejor recuperación física, sino que además debe incluir el análisis rutinario de tóxicos y actuaciones tendentes a prevenir la reincidencia (prevención secundaria). Una intervención psicológica breve se ha mostrado eficaz en este sentido.

\section{Declaración de conflicto de intereses}

Los autores han declarado no tener ningún conflicto de intereses.

\section{BIBLIOGRAFÍA}

1. Lundberg GD. Ethyl alcohol: ancien plague and modern poison. JAMA. 1984;252:1911-2.

2. Rivara FP, Jurkovich GJ, Gurney JF. The magnitude of acute and chronic alcohol abuse in trauma patients. Arch Surg. 1993; 128:907-13.

3. Del Río C, Gómez J, Sancho M, Álvarez FJ. Alcohol, illicit drugs and medicinal drugs in fatally injuried drivers in Spain between 1991 and 2000. Forensic Sci Int. 2002;127:63-70.

4. Díaz R, Aguilar E, Herrera L, Carbona S, Guerrero F, Fernández-Mondéjar E. Presencia de tóxicos en pacientes traumatizados graves. Med Intensiva. 2005;29 (Extraordinario 1):61.

5. Soderstrom CA, Dischinger PC, Kerns TJ, Kufera JA, Mitchel KA, Scalea TM. Epidemic increases in cocaine and opiate use by trauma center patients: Documentation with a large clinical toxicology database. J Trauma. 2001;51:557-64.

6. Walsh J, Flegel R, Cangianelli, Atkins R, Soderstrom C, Kerns T. Epidemiology of alcohol and other drug use among motor vehicle crash victims admitted to a trauma center. Traffic Inj Prev. 2004;5:254-60.

7. Los andaluces ante las drogas. Consejería de Igualdad y Bienestar Social; 2005. p. 67-75.

8. Dischinger PC, Mitchel KA, Kufera JA, Soderstrom CA, Lowenfels AB. A longitudinal study of former trauma center patients: the assotiation between toxicology status and subsequent injury mortality. J Trauma. 2001;51:884-6.

9. Hungerford DW. Recommendation for trauma centers to improve screening, brief intervention, and referral to treatment for substance use disorders. J Trauma. 2005;59:S37-S42.

10. Gentilello LM, Rivara FP, Donovan DM, Jurkovich GJ, Daranciang E, Dunn CW, et al. Alcohol interventions in a trauma center as a means of reducing the risk of injury recurrence. Ann Surg. 1999;230:473-80.

11. Zatzick D, Roy-Birne P, Russo J, Rivara F, Droesch R, Wagner A, et al. A randomized effectiveness trial of stepped collaborative care for acutely injured trauma survivors. Arch Gen Psychiatry. 2004;61:498-506.

12. Gentilello LM. Alcohol and injury. American College of surgeons committee on trauma Requeriments for trauma center intervention. J Trauma. 2007;62:S44-S45. 